\title{
EL CONTENIDO CERÁMICO DE UNA ESTRUCTURA ALFARERA EN LA DEPRESIÓN DE RONDA (S. V A.C.): ANÁLISIS E INTERPRETACIÓN
}

\author{
CERAMIC CONTENT OF A POTTERY STRUCTURE IN THE DEPRESSION \\ OF RONDA (5TH BC): ANALYSIS AND INTERPRETATION
}

\author{
CLAUDIA SANNA* \\ ELENA CAPPAI** \\ MARGHERITA DEMONTIS**
}

\begin{abstract}
Resumen: La Depresión de Ronda (Málaga, España) es una comarca montañosa y periférica con respecto al núcleo central donde se investiga la caracterización material de la cultura arqueológica "turdetana". Por otro lado, con una personalidad propia derivada de contactos multiétnicos, constituye un punto clave para examinar las comunidades indígenas del interior de los sistemas béticos. El objetivo de este trabajo es el análisis y la interpretación del contenido arqueológico de un horno cerámico bicameral hallado en Ronda ciudad, que se llevó a cabo gracias a la documentación de la excavación, el buen estado de conservación y el proceso de restauración de los materiales. Además, se plantean algunas cuestiones sobre las dinámicas productivas locales y sus correlatos sociales y políticos rastreables al comienzo de la II Edad del Hierro. Palabras Clave: Turdetania, alfarería, análisis arqueológico, II Edad del Hierro
\end{abstract}

\footnotetext{
* Doctoranda del Departamento de Prehistoria y Arqueología de la Universidad de Granada. Campus Cartuja s/n, 18012, Granada. Correo-e: csanna_1@ugr.es
}

\begin{abstract}
The Depression of Ronda (Malaga, Spain) is a mountainous region peripheral to the central nucleus where material characterization of "Turdetan" archaeological culture is investigated. On the other hand, with its own personality derived from multi-ethnic contacts, it is a key point to examine the indigenous communities from the interior of the Baetic System. The aim of this study is to analyse and interpret the archaeological content of a bicameral ceramic kiln found in the town of Ronda, carried out thanks to the documentation of excavation, a good state of conservation and restoration process. In addition, some issues on local production dynamics as well as their social and political correlates traceable at the beginning of the Middle Iron Age are raised.
\end{abstract}

Keywords: Turdetania, pottery, archaeological analysis, Middle Iron Age 


\section{EL MARCO GEOGRÁFICO DE ESTUDIO Y SU RELACIÓN CON LA TURDETANIA}

En el marco de la formación de la cultura ibérica del Suroeste peninsular, las diversas publicaciones científicas han hecho hincapié sobre todo en las transformaciones socio-económicas y políticas rastreables arqueográficamente en los oppida indígenas, considerados epónimos, dejando a un lado, bien porque se consideren subsidiarios de estos bien porque una de las prioridades ha sido la búsqueda de una homogeneización "regional" (Turdetanos, Bastetanos, etc.), las comunidades locales del interior y sus dinámicas internas. Desde los años noventa, en la Depresión de Ronda se han venido publicando una serie de estudios sobre la cuestión del surgimiento de una sociedad centralizada y jerarquizada a partir del siglo VI a.C. (Carrilero 1992), definida genéricamente como ibérica, pero que en lo específico correspondería para algunos con comunidades celtas y para otros con turdetanos, herederos del sustrato tartésico (Ferrer y García Fernández 2008: 201). Recientemente E. Ferrer y F. J. García (2002: 149; García Fernández 2012: 703-704) han remarcado el carácter multiétnico de la Turdetania (el concepto de etnia se utiliza a lo largo de todo el artículo con el significado expresado en Carrilero y Aguayo 2008: 181), lo que según nuestro planteamiento permite superar las fronteras levantadas a partir de la búsqueda de unas correspondencias forzadas entre textos clásicos y material arqueológico, y tener en cuenta las peculiaridades locales/comarcales identificables en este amplio territorio, correspondientes a varias comunidades que se han relacionado, confrontado y enfrentado en continuas negociaciones de poder, a lo largo de todo el I milenio a.C. (véase para la cuestión sobre el apelativo ibérico dado a la cerámica turdetana: Escacena 1989, y para la puesta en duda de la cerámica como indicador étnico: Escacena 1992).

La posición geográfica ocupada por la Depresión de Ronda la sitúa entre las zonas óptimas para analizar los posibles fenómenos de hibridación que pudieron caracterizar las relaciones culturales ocurridas entre las diferentes comunidades que poblaban su entorno, y que a lo largo del proceso, iniciado en el siglo VIII a.C., dieron lugar a la conformación de la sociedad primero cilbicena (véase el desarrollo y aplicación del término en Carrilero y Aguayo 2008) y más tarde "turdetana" (?) de la zona (nos referimos de esta manera a los habitantes de la Depresión de Ronda, incluidos posiblemente en una región geográfica multiétnica y con fronteras no fijas y permeables).Con el vocablo "turdetano", escrito entre comillas, pretendemos resaltar el amplio debate existente sobre su uso (véase: Ferrer y García 2002, Ferrer y García 2008, García 2012). De hecho, la Depresión está conectada tanto con la costa atlántica a través del río Guadalete, que antiguamente desembocaba en una bahía junto al asentamiento fenicio del Castillo de Doña Blanca, como con la costa mediterránea, pasando por los ríos y arroyos que constituyen la cabecera del río Guadalhorce, que desagua muy próximo al asentamiento fenicio del Cerro del Villar (fig. 1).

Las campañas de prospecciones y excavaciones arqueológicas realizadas en los principales asentamientos de la Depresión de Ronda, Ronda la Vieja (Acinipo), Ronda ciudad y Silla del Moro, y en su entorno geográfico han permitido acrecentar la información disponible para la época "turdetana", aunque de una parte el carácter preventivo de la mayoría de estas investigaciones, en el caso de Ronda ciudad, y de otra el retraso en la publicación de todo el conjunto de datos arqueográficos, hacen que el conocimiento sobre el paso entre la I y la II Edad del Hierro siga siendo fragmentario. Estudios recientes han ido ampliando la documentación específica y se han dirigido sobre todo al análisis de los procesos políticos, sociales y económicos desde diferentes perspectivas (Aguayo et al. 2013; Aguayo y Sanna 2014; Sanna y Aguayo en prensa).

En particular, uno de los ámbitos que deben ser abordados es la cuestión de la organización económica de estas comunidades, a partir de los centros productivos como los talleres alfareros (véase a este propósito Sáez 2013), que lejos de ser considerados parte de la base de la economía de estas comunidades, debían más bien tener un papel importante en las manifestaciones y mantenimiento de las relaciones de poder.

El principal objetivo de este estudio es, por lo tanto, presentar el análisis del contenido cerámico de la piroestructura hallada en Ronda ciudad, para tener un marco descriptivo y analítico, aunque parcial, de la producción alfarera en la Depresión, y luego plantear algunas cuestiones, a profundizar en futuros estudios, especialmente dirigidas al conocimiento de las dinámicas productivas y distributivas de cerámicas entre las poblaciones serranas "turdetanas" y sus relaciones con los hábitos de consumo. 


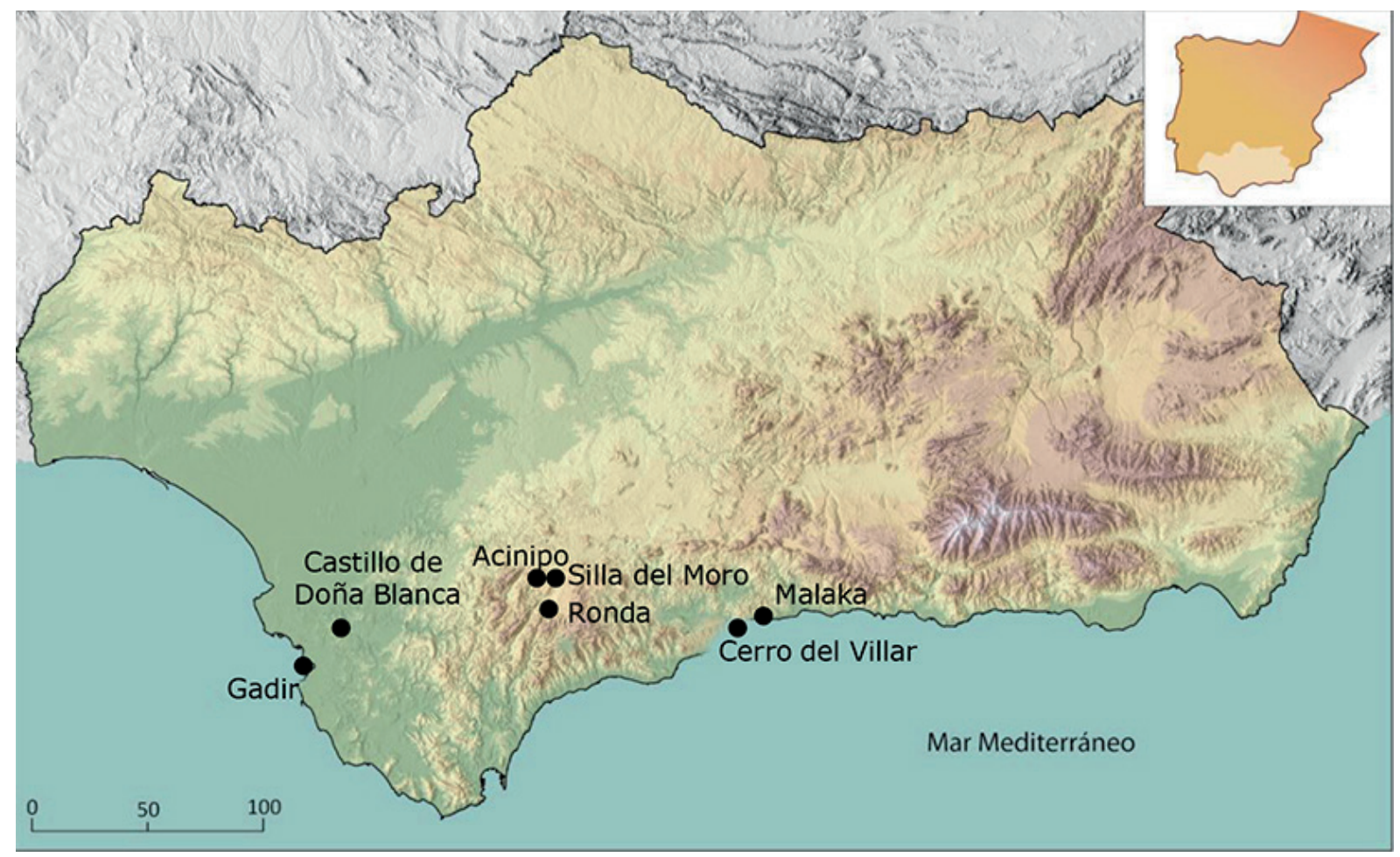

Figura 1. Mapa de Andalucía con los tres principales yacimientos de la Serranía de Ronda y los asentamientos más destacados de las costas andaluzas (realizado por D. García González y modificado por C. Sanna).

\section{ANÁLISIS E INTERPRETACIÓN DE LOS USOS DE LA PIROESTRUCTURA A TRAVÉS DE SU COLMATACIÓN ARQUEOLÓGICA}

El descubrimiento del horno alfarero de Ronda ciudad se debe a una excavación de urgencia realizada antes de la edificación de un solar que se extiende entre las calles Armiñán no 39, 41 y 43 y Aurora ${ }^{\circ} 16$ en 1989 (Aguayo et al. 1992: 339-341) (fig. 2). Su técnica de realización, consistente en la excavación de una profunda fosa en la cual disponer la cámara de combustión, ha hecho que sobre todo esta última haya sido hallada en buen estado de conservación, junto con el pilar central y parte del praefurnium (Aguayo et al. 2013: 144-145; para un estado de la cuestión sobre los hornos bicamerales protohistóricos y romanos hallados en el Valle del Guadalquivir véase: García Fernández y García Vargas 2012).

La cámara inferior estaba conformada por ladrillos de adobe $(30 \times 30 \times 10 \mathrm{~cm})$, dispuestos $a$ soga. Otros dos ladrillos (60 × $60 \times 10 \mathrm{~cm})$, también de adobe, constituían el límite del praefurnium y estaban colocados enfrentados en sentido vertical, con una separación de $56 \mathrm{~cm}$. Por último, el pilar central, siempre de ladrillos de adobe y revocado, medía $95 \mathrm{~cm}$ de longitud por $25 \mathrm{~cm}$ de anchura (Aguayo et al. 2013: 146).

Las óptimas condiciones del hallazgo y el escaso conocimiento de piroestructuras en los años ' 80 , llevó a los arqueólogos a elegir un método de excavación meticuloso, procediendo a la división del área a excavar en dos mitades, la primera, que de ahora en adelante indicaremos como H1, que va del praefurnium hasta el pilar central, y la segunda, desde ahora $\mathrm{H} 2$, desde el pilar central hasta la pared del fondo (fig. 3). Esta subdivisión del área excavada se refleja también en el material cerámico que ha sido organizado en lotes estratigráficos, correspondientes unos a $\mathrm{H} 1$ y otros a $\mathrm{H} 2$. Por lo tanto, una de las prioridades del análisis cerámico ha sido la de identificar los lotes correspondientes a las dos mitades del horno y homogeneizar los estratos identificados, a partir de la consulta de toda la documentación relativa a la excavación arqueológica -informes de los materiales hallados, plantas y perfiles de la estructura-.

La metodología aplicada al estudio de este material cerámico responde a los dos objetivos principales propuestos: identificar, en lo posible, las diferentes fases de utilización del horno bien como piroestructura bien 


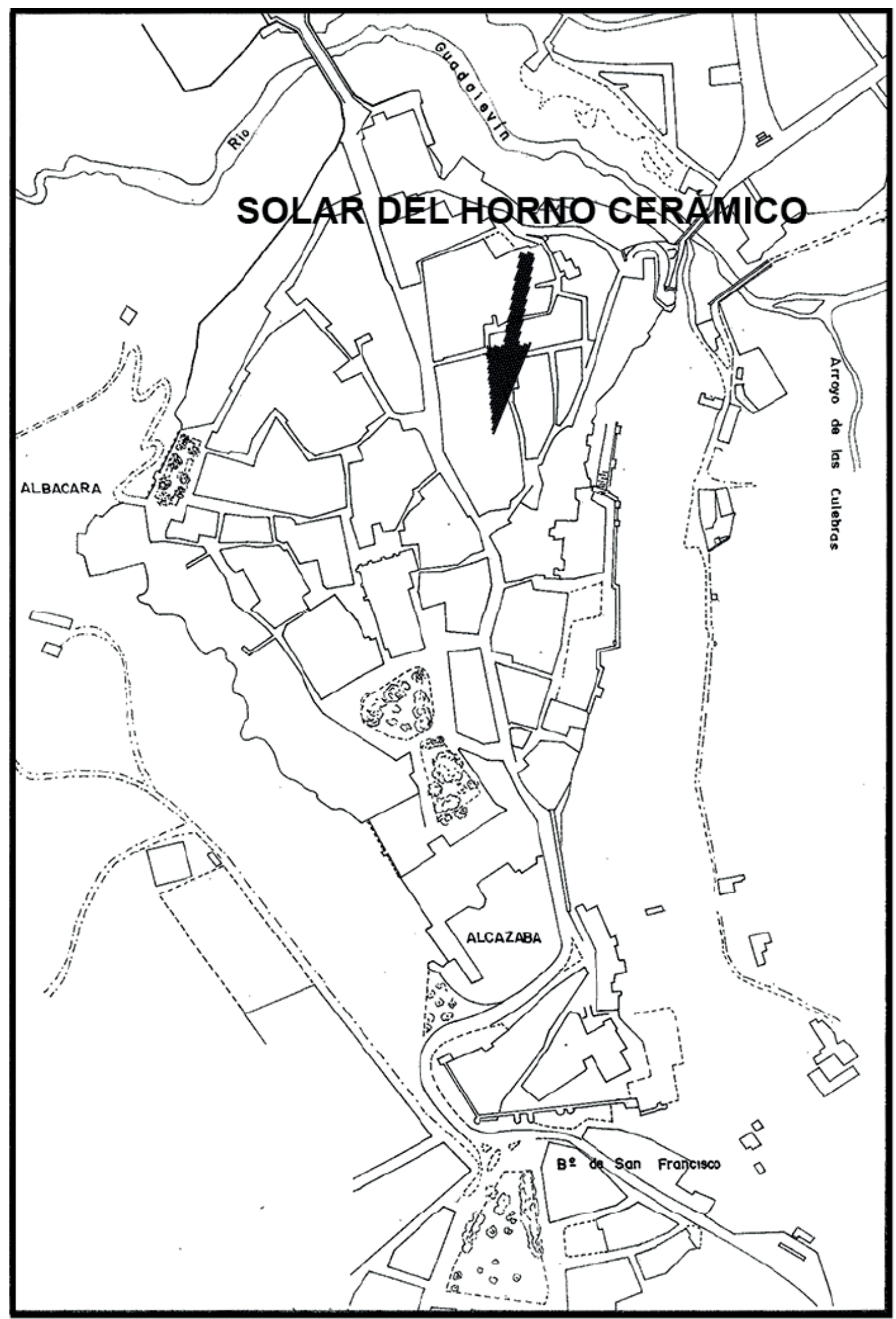

Figura 2. Plano del casco antiguo de Ronda ciudad con la localización del horno cerámico. 


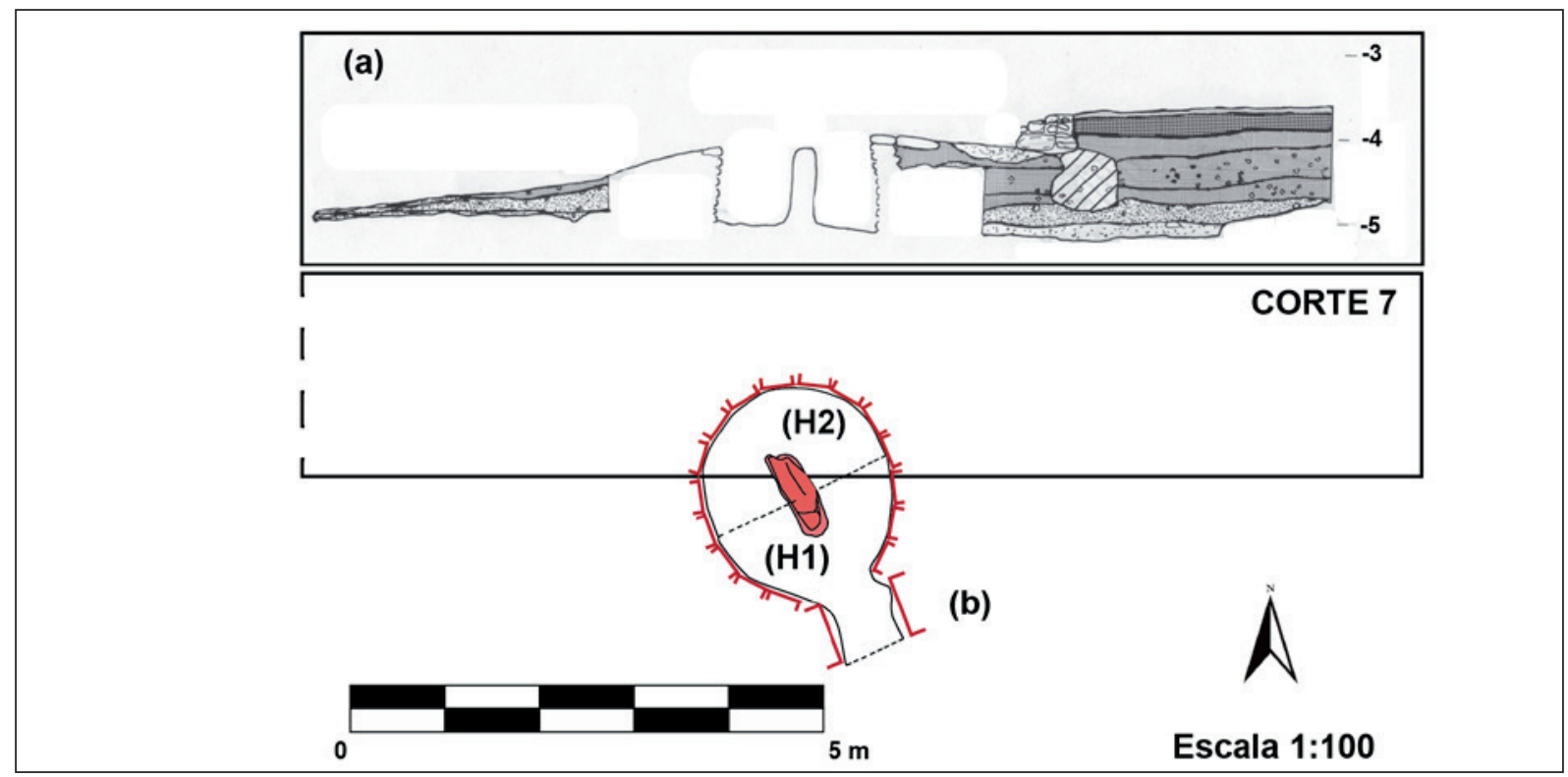

Figura 3. Perfil sur del corte 7 (a) y planta del horno cerámico en relación al mismo corte (b).

como basurero, a través de la identificación de la vajilla propia de cada momento, y determinar el tiempo de su funcionamiento y amortización. A este propósito nos hemos remitido a las pautas indicadas por J. Thiriot (1992: 144-145): estudio del conjunto de informaciones disponibles para hornos a examen (estratigrafías, morfologías, tecnologías y tipos de pastas cerámicas, entornos arqueológicos) y la meticulosidad metodológica en el momento de diferenciar las distintas fases de utilización y colmatación de la piroestructura (producción, abandono, reutilización, derrumbe y amortización).

El primer paso ha sido pedir la colaboración de dos especialistas en restauración para manejar con el mayor cuidado posible estas piezas que, además de haber estado guardadas en cajas y almacenadas a lo largo de unos 25 años, necesitaban tratamientos específicos para reducir y/o eliminar las incrustaciones y no dañar ni las superficies ni las eventuales decoraciones, muy deterioradas no solo por los naturales procesos posdeposicionales, sino también por el escaso control mostrado por los alfareros "turdetanos" en las diferentes fases de producción. Mientras el procedimiento de consolidación será explicado en un apartado específico más adelante, podemos aquí ya adelantar que a través de este meticuloso trabajo se han podido identificar y pegar las piezas cerámicas reconstruibles y corregir la adjudicación de algunos materiales, trasladándolos al nivel correspondiente. Además, el trabajo en equipo ha permitido relacionar con mayor seguridad parte de los lotes de las dos zonas del horno, y llegar a una buena precisión estratigráfico-funcional.

Posteriormente, se ha procedido a documentar gráficamente todas las piezas, para poder realizar una primera clasificación tipológico-funcional. Para ello se ha considerado oportuno, con el objetivo de una necesaria y deseable tipología local/comarcal de las cerámicas "turdetanas", proceder a las mediciones del diámetro y al EVE de cada fragmento, y determinar el color de las piezas a través del uso de las tablas Munsell. En particular, el EVE ("Estimated vessel equivalent" o sea el equivalente de vasija estimado) es un criterio de cuantificación de la cerámica que permite calcular el porcentaje conservado de la vajilla a partir de un fragmento del borde y/o de la base, siempre que se conozca el diámetro. De este modo se puede calcular por cada tipo cerámico el porcentaje estimado de conservación.

La inexistencia de una tipología general de las variadas formas del Suroeste peninsular (Ferrer y García Fernández 2008: 207-212), en parte justificada por la monotonía de las mismas a lo largo de toda la II Edad del Hierro, y la imposibilidad de realizar una propia de la Depresión dada la escasez del repertorio cerámico y la fragmentación de la muestra encontrada en el horno de Ronda ciudad, nos ha hecho optar por una clasificación funcional que contemple cuatros grupos principales: de cocina, de mesa, de transporte/almacenamiento y de otros usos -donde hemos incluido los cuencos/lucernas-, a partir de la comparación con diversas tipologías 
Tabla 1. Correspondencia entre los lotes de H1 y H2, y sucesión estratigráfica.

\begin{tabular}{|c|c|c|c|c|c|c|c|}
\hline \multicolumn{4}{|c|}{$\mathrm{H} 1$} & \multicolumn{4}{|c|}{$\mathrm{H} 2$} \\
\hline $\begin{array}{c}\text { Unidad } \\
\text { Estratigráfica } \\
\text { (UE) }\end{array}$ & $\begin{array}{c}\text { Profundidad } \\
\text { Alcanzada }\end{array}$ & $\begin{array}{l}\text { Nivel del } \\
\text { Hallazgo }\end{array}$ & $\begin{array}{c}\text { Lote } \\
\text { Cerámico }\end{array}$ & $\begin{array}{c}\text { Unidad } \\
\text { Estratigráfica } \\
\text { (UE) }\end{array}$ & $\begin{array}{c}\text { Profundidad } \\
\text { Alcanzada }\end{array}$ & $\begin{array}{l}\text { Nivel del } \\
\text { Hallazgo }\end{array}$ & $\begin{array}{c}\text { Lote } \\
\text { Cerámico }\end{array}$ \\
\hline UE 1 y 2 & $\begin{array}{l}\text { De }-4.30 m \\
\mathrm{a}-4.80 \mathrm{~m}\end{array}$ & Derrumbe & 7461 & UE 1 y 2 & $\begin{array}{l}\text { De }-4.30 \mathrm{~m} \\
\mathrm{a}-5.00 \mathrm{~m}\end{array}$ & Derrumbe & 7538 \\
\hline UE 3 y 4 & $\begin{array}{l}\text { De }-4,80 m \\
a-5.10 m\end{array}$ & Basurero & $\begin{array}{l}7499 \\
7501\end{array}$ & UE 3 & $\begin{array}{l}\text { De }-5.00 m \\
a-5.15 m\end{array}$ & Basurero & $\begin{array}{l}7546 \\
7560 \\
7575\end{array}$ \\
\hline UE 5 y 6 & $\begin{array}{l}\text { De }-5,10 m \\
a-5.25 m\end{array}$ & $\begin{array}{l}\text { Basurero/ } \\
\text { Fallos de cocción }\end{array}$ & 7526 & UE 4 & $\begin{array}{l}\text { De }-5.15 \mathrm{~m} \\
\mathrm{a}-5.25 \mathrm{~m}\end{array}$ & $\begin{array}{l}\text { Basurero/ } \\
\text { Fallos de cocción }\end{array}$ & $\begin{array}{l}7570 \\
7565\end{array}$ \\
\hline \multicolumn{4}{|c|}{ Nivel de ánforas (7533) } & \multicolumn{4}{|c|}{ Nivel de ánforas ausente } \\
\hline UE 7 & $\begin{array}{l}\text { De }-5.25 \mathrm{~m} \\
\text { a roca madre }\end{array}$ & Fallos de cocción & 7536 & UE 7 & $\begin{array}{l}\mathrm{De}-5.25 \mathrm{~m} \\
\text { a roca madre }\end{array}$ & Fallos de cocción & 7599 \\
\hline
\end{tabular}

realizadas por distintos autores (Aubet et al. 1999; Belén 2006; Escacena 1979-1980; Escacena 2001; Ferrer y García Fernández 2008; Mata y Bonet 1992; Pellicer 1978; Pereira 1988; Pereira 1989; Ramón 1995; Ramón et al. 2007), que ha servido no solo para utilizar una terminología específica y lo más homogénea posible, sino también para afinar la cronología y encontrar parecidos entre nuestros tipos y los procedentes de otros asentamientos andaluces.

El horno alfarero de la ciudad de Ronda ha conservado una estratigrafía de un metro de potencia. Tanto en $\mathrm{H} 1$ como en $\mathrm{H} 2$, a través del proceso de excavación, del análisis del material y del trabajo de restauración, ha sido posible diferenciar hasta 4 fases distintas de utilización del horno, constituidas por (de abajo hacia arriba): fallos de cocción, mezcla de fallos de cocción y basura, solo basura y derrumbe (tabla 1).

El nivel superior, interpretado como derrumbe, alcanza unos $50 \mathrm{~cm}$ de grosor en la parte $\mathrm{H} 1$ y $70 \mathrm{~cm}$ en la H2, debido a una mayor potencia de este nivel en la parte posterior del horno (tabla 2). El material arqueológico está formado por los adobes alargados de la criba, parte de los ladrillos de sección rectangular que formaban la cámara de cocción y poca vajilla entremezclada y muy fragmentaria. La mayoría de las cerámicas pertenecen a formas abiertas asociadas al repertorio doméstico, representadas sobre todo por cuencos de labio pintado y borde indiferenciado (véase como ejemplo RO'89-AA/7538-4, fig. 4 c). Las únicas piezas que sobresalen por su alto porcentaje de conservación son respectivamente un vaso globular y pintado (RO'89/7461-8 y 7499-9, fig. 4 e), cuyo perfil es arqueológicamente completo teniendo el 60\% de EVE del labio (Orton et al. 1997: 194-197), y un lebrillo (RO'89/7461-9 y 7499-10) del que se conserva el 40\%. Después del paciente trabajo de lavado, limpieza y búsqueda de las piezas colindantes, se ha observado que ambos ejemplares en realidad están formados por fragmentos que pertenecen a dos unidades distintas y asignadas, en fase de excavación, a dos diferentes niveles artificiales, como son la 7461 del mismo derrumbe y la 7499 del nivel de basura (tabla 1). Se ha detectado, por lo tanto, la presencia de claras mezclas cerámicas entre un estrato arqueológico y otro, hecho muy común cuando se excava por alzadas artificiales y además en un contexto arqueológico como un horno que, aunque sea cerrado en origen, ha sufrido diferentes momentos de amortización y de natural desprendimiento de la estructura sobreelevada. A través del análisis macroscópico de las cerámicas, con el fin de establecer el estado y el porcentaje de conservación de las piezas, la presencia o ausencia de fallos de cocción y finalmente la comparación con el material de ambos niveles, se considera que las dos vajillas pertenecen al nivel inmediatamente inferior, el interpretado como basurero. De hecho, tanto el vaso globular como el lebrillo no presentan prácticamente evidencias de fallos de cocción si se exceptúan, en la pared del lebrillo, algunas "burbujas" debidas probablemente a una cantidad excesiva de carbonatos visibles macroscópicamente en la misma pasta cerámica y que en algún caso llegaron a estallar. De todos modos estas "burbujas" no parecen haber comprometido la 
funcionalidad de la pieza, que fue probablemente arrojada en el horno después de un tiempo de utilización y natural rotura.

Tabla 2. Cuantificación del material cerámico hallado en el derrumbe y su distribución espacial.

\begin{tabular}{|l|c|c|}
\cline { 2 - 3 } \multicolumn{1}{c|}{} & \multicolumn{2}{c|}{ Nivel de derrumbe } \\
\cline { 2 - 3 } \multicolumn{1}{c|}{} & H1 & H2 \\
\hline Formas abiertas & 4 & 14 \\
\hline Formas cerradas & 0 & 4 \\
\hline Bordes & 2 & 15 \\
\hline Fondos & 2 & 3 \\
\hline Asas & 0 & 0 \\
\hline NMI & 4 & 15 \\
\hline Amorfos remontables & 5 & 14 \\
\hline Cocina & 0 & 1 \\
\hline Cerámica de mesa & 4 & 17 \\
\hline Transporte/almacenamiento & 0 & 0 \\
\hline Otros usos & 0 & 0 \\
\hline Mano & 0 & 1 \\
\hline Torno & 4 & 17 \\
\hline Total fragmentos diagnósticos & & 22 \\
\hline
\end{tabular}

Justo por debajo de este primer nivel, nos encontramos con el segundo, que corresponde plenamente a la fase de amortización del horno y que tiene una potencia de unos 30 centímetros en $\mathrm{H} 1$ y unos 15 centímetros en $\mathrm{H} 2$, de modo que a partir de ahora el plano del fondo resulta igualado en su totalidad (tabla 3 ). El abundante material cerámico, netamente superior a los otros tres niveles identificados, estaba englobado en sedimentos de color marrón grisáceo muy sueltos, que contenían carbones y cenizas distribuidas en manchas discontinuas. Las características principales que nos sugieren la utilización como basurero doméstico de este nivel arqueológico de la piroestructura son el alto porcentaje de conservación de las piezas, la casi ausencia de huellas referibles a fallos de cocción y la representatividad de las cuatro categorías funcionales utilizadas, aunque la cerámica de mesa sea predominante.

Entremezclado con este material, hay que destacar la vasija RO'89/7546-9 (fig. 4 g), parcialmente reconstruible, y que por su complejo sistema decorativo -bandas anchas y rojas entre líneas negras, semicírculos, aguadas, cuartos de aspa y grupos de tres líneas paralelas de color negro-, no parece responder estrictamente a un uso cotidiano. Además, la presencia numéricamente destacable de otras paredes de vasos similares, todos pintados con motivos decorativos parecidos $\mathrm{y}$ procedentes del nivel de basura, podría indicarnos su posible producción en una piroestructura cercana.

Tabla 3. Cuantificación del material cerámico hallado en el basurero y su distribución espacial.

\begin{tabular}{|l|r|r|}
\cline { 2 - 3 } \multicolumn{1}{c|}{} & \multicolumn{2}{c|}{ Nivel de basurero } \\
\cline { 2 - 3 } \multicolumn{1}{c|}{} & H1 & H2 \\
\hline Formas abiertas & 10 & 36 \\
\hline Formas cerradas & 3 & 6 \\
\hline Bordes & 13 & 34 \\
\hline Fondos & 1 & 8 \\
\hline Asas & 0 & 2 \\
\hline NMI & 13 & 35 \\
\hline Amorfos remontables & 13 & 26 \\
\hline Cocina & 0 & 1 \\
\hline Cerámica de mesa & 10 & 37 \\
\hline Transporte/almacenamiento & 0 & 2 \\
\hline Otros usos & 1 & 2 \\
\hline Mano & 2 & 1 \\
\hline Torno & 9 & 41 \\
\hline Total fragmentos diagnósticos & & 56 \\
\hline
\end{tabular}

El conjunto de material hallado en este nivel parece encuadrable cronológicamente en el siglo $\mathrm{V}$ a.C. a partir sobre todo de los motivos decorativos y el acabado del fondo, por medio de espatulado, observable tanto en la forma pintada RO'89/7546-9 (fig. 4 g) como en el vaso globular RO'89/7461-8 y 7499-9 (fig. 4 e). La única pieza que se aleja de esta fecha es el ánfora RO'89/7546-6 y 7560 (fig. 4 f) (paralelos en Pellicer 1978: 383, fig. 6, n.1637, que la data a principios del siglo III a.C.). La presencia de este único fragmento, relacionable con una prosecución de la fase de amortización del horno hasta por lo menos inicios del siglo III a.C., y la misma naturaleza inestable de la estructura alfarera, que ya de por sí limita el período de su colmatación, nos sugiere que se trate más bien de una intrusión. 
El tercer nivel estratigráfico se compone de un paquete de unos 15 centímetros de potencia, conteniendo una cantidad de cerámica menor "al nivel anterior", muy fragmentada, y donde aparecen las primeras cerámicas claramente pasadas de cocción (tabla 4). A nivel estratigráfico se ha añadido el posible borde pintado de una forma cerrada, la RO'89/7599-14, que en fase de excavación había sido incluido de manera incorrecta en el último lote, el de los fallos de cocción.

Numéricamente prevalecen los cuencos de borde indiferenciado y pintado en el labio, algunos claramente fallos de cocción que estarían indicando una de las producciones más típicas de esta zona artesanal, pudiendo relacionarlos más probablemente con piroestructuras cercanas y contemporáneas (Aguayo et al. 2013: 151-152).

Tabla 4. Cuantificación del material cerámico hallado en el nivel de basurero/fallos de cocción y su distribución espacial.

\begin{tabular}{|l|c|c|}
\cline { 2 - 3 } \multicolumn{1}{c|}{} & \multicolumn{2}{c|}{$\begin{array}{c}\text { Nivel de basurero/ } \\
\text { fallos de cocción }\end{array}$} \\
\cline { 2 - 3 } \multicolumn{1}{c|}{ Formas abiertas } & H1 & H2 \\
\hline Formas cerradas & 1 & 7 \\
\hline Bordes & 8 & 1 \\
\hline Fondos & 1 & 0 \\
\hline Asas & 0 & 0 \\
\hline NMI & 8 & 7 \\
\hline Amorfos remontables & 4 & 10 \\
\hline Cocina & 1 & 0 \\
\hline Cerámica de mesa & 7 & 8 \\
\hline Transporte/almacenamiento & 1 & 0 \\
\hline Otros usos & 0 & 0 \\
\hline Mano & 1 & 0 \\
\hline Torno & 8 & 7 \\
\hline Total fragmentos diagnósticos & & 17 \\
\hline
\end{tabular}

La fecha de la segunda mitad del siglo V a.C., obtenida a través de una datación radiocarbónica calibrada (Aguayo et al. 2013: 148) y que haría referencia a la época de funcionamiento del horno como piroestructura, parece avalada en este mismo nivel por el hallazgo del borde de un ánfora, la RO'89/7526-13 (fig. 4 d), que por sus características es asimilable a los envases de Cerro Macareno (Pellicer 1978: 372-377, fig. 3, n. 994, de mediados del s. VI, y la n. 938, de principios del siglo VI que, en tipos evolucionados, perdura hasta el V a.C.). Solamente en la mitad anterior del horno, H1, y localizadas en un espacio concreto, es decir a la izquierda de la entrada con respecto al praefurnium, se han encontrado fragmentos de paredes de ánforas, dispuestos unos al lado de los otros formando lo que se ha interpretado como un suelo o mejor un parche solado con material refractario. Alrededor de estas ánforas y en la otra mitad del horno, se ha individuado el último nivel arqueológico, de unos 15 centímetros de potencia, que llega hasta la roca madre (tabla 5). Las cerámicas son casi todas fallos de cocción clarísimos, entre los cuales sobresalen dos cuencos, uno de borde engrosado y pintado, cuyo borde está casi completo (RO'89AA/7599-7, fig. 4 a) y otro con evidentes huellas de fallos de cocción (RO’89-AA/7599-4-3, fig. 4 b).

Tabla 5. Cuantificación del material cerámico hallado en el nivel de fallos de cocción y su distribución espacial.

\begin{tabular}{|c|c|c|}
\hline & \multicolumn{2}{|c|}{$\begin{array}{l}\text { Nivel de fallos } \\
\text { de cocción }\end{array}$} \\
\hline & $\mathrm{H} 1$ & $\mathrm{H} 2$ \\
\hline Formas abiertas & 1 & 6 \\
\hline Formas cerradas & 0 & 2 \\
\hline Bordes & 1 & 8 \\
\hline Fondos & 0 & 0 \\
\hline Asas & 0 & 0 \\
\hline NMI & 1 & 9 \\
\hline Amorfos remontables & 1 & 0 \\
\hline Cocina & 0 & 0 \\
\hline Cerámica de mesa & 1 & 1 \\
\hline Transporte/almacenamiento & 0 & 0 \\
\hline Otros usos & 0 & 0 \\
\hline Mano & 0 & 0 \\
\hline Torno & 1 & 9 \\
\hline Total fragmentos diagnósticos & & \\
\hline
\end{tabular}

La disposición in situ de muchos de estos fragmentos, la ausencia de fondos, el aspecto rodado de 


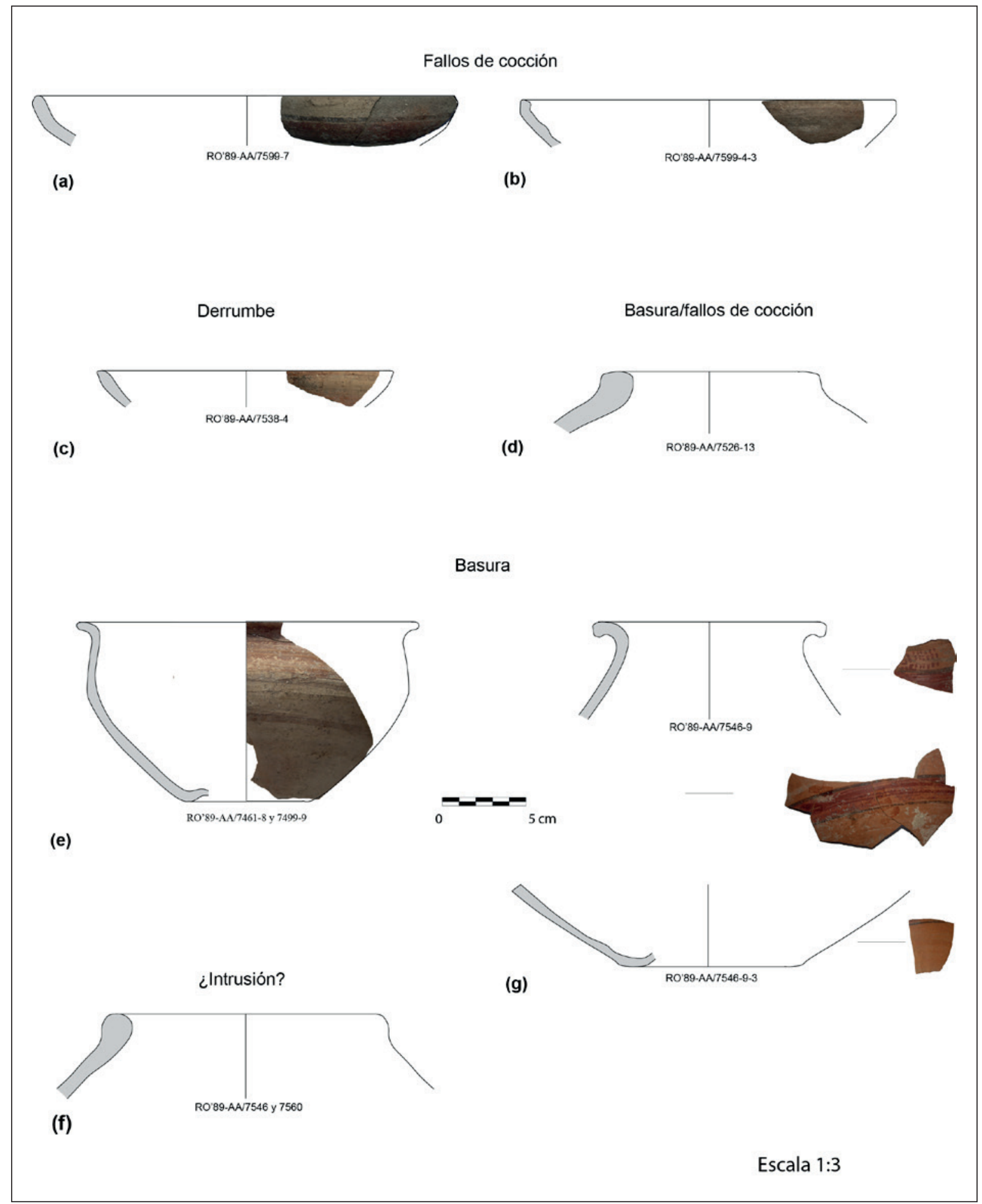

Figura 4. Selección de las principales formas cerámicas halladas en el horno de Ronda ciudad. 
algunos, nos sugiere que se trata de piezas mal cocidas en este mismo horno, o en otros que debían estar en las proximidades, y que fueron reutilizadas para reparar el mismo fondo de la cámara de combustión o recalzar las paredes.

\section{VALORACIONES Y CONSIDERACIONES ARQUEOLÓGICAS CONTEXTUALES E INTERPRETATIVAS}

El horno cerámico de Ronda ciudad, por el buen estado de conservación en el cual se ha hallado, nos permite hacer una serie de observaciones sobre el sistema productivo "turdetano" y sus implicaciones sociales.

Primero las asignaciones tipológicas y cronológicas del material cerámico analizado vienen a confirmar las dos fechas radiocarbónicas referidas: la más antigua del nivel arqueológico previo a la construcción y uso del horno, con una datación del siglo VII a.C., y la otra referible al combustible de la última hornada, que según la calibración directa ocurrió en el 432 a.C. (Aguayo et al. 2013: 148). A ello hay que añadir el hallazgo de algunas cerámicas áticas cercanas al área investigada, que también han sido datadas en la segunda mitad del siglo V o primera mitad del IV a.C. De hecho, la aparición del borde de ánfora RO'89/7526-13 (fig. 4 d) entre los fallos de cocción documentados en el tercer nivel estratigráfico viene a confirmar esta atribución cronológica. Otro importante dato temporal procede del nivel de amortización, en el cual encontramos el vaso globular RO'89/7461-8 y 7499-9 (fig. 4 e) y el pintado RO'89/7546-9 (fig. 4 g) pertenecientes, por acabado y decoración, al siglo $\mathrm{V}$ a.C.

El número total de fragmentos cerámicos analizados es, entre diagnósticos y amorfos restituibles, de 177, de los cuales 95, o sea más de la mitad, proceden del nivel de amortización (tabla 3). Nuestra interpretación de este nivel es que se trataría de un depósito de basura doméstica, por la representatividad de casi todo el ajuar doméstico -incluidas ollas y posibles lucernas también a mano- y la casi ausencia de fallos de cocción, lo que nos indica una frecuentación de esta zona relativamente intensa y continuada en el tiempo. Los principales generadores de estos desechos serían los grupos familiares de los mismos alfareros que debían habitar en el propio taller cerámico.

Además la presencia, aunque escasa, de cerámicas mal cocidas en todos los niveles de la piroestructura demuestra claramente la continuidad de la actividad alfarera en las proximidades. En efecto, era práctica común en los talleres artesanales, como parece ser este de Ronda ciudad, construir hornos unos cerca de otros, con la doble funcionalidad de reducir los esfuerzos productivos y de ir aprovechando todos los espacios del taller, como le ocurrió al horno objeto de estudio, que una vez inutilizado como estructura alfarera, fue reaprovechado como basurero hasta que, probablemente, se derrumbaron las paredes del laboratorio y la criba/solera. A este propósito, la misma diferencia de concentración del material entre un sector y otro del horno podría explicarse por la ruptura parcial del suelo de la cámara de cocción o criba/solera en la parte $\mathrm{H} 2$, motivo que impidió seguir utilizando el horno como tal; de hecho, en el mismo nivel de fallos de cocción hay una cantidad de material cerámico hasta seis veces superior a la de H1, lo que hace suponer que pudo haber sido la parte más débil de la piroestructura también en su última (?) hornada (tabla 5). No es de excluir tampoco, que esta diferencia de proporción entre $\mathrm{H} 1$ y $\mathrm{H} 2$ se deba a la mayor cercanía de la parte posterior del horno a otras estructuras del taller, siendo más práctico verter el material de desecho en este lado. El mismo peso de estos desechos habría causado primero la ruptura de las superestructuras de $\mathrm{H} 2$ y sólo en un momento posterior se habrían derrumbado también las correspondientes a H1.

Un tema abierto y de gran interés para conocer el probable proceso de hibridación que llevó a la formación de la cultura material "turdetana" está representado por la misma cerámica. La preeminencia de los estudios tipológicos de las cerámicas foráneas, sobre todo fenicias o griegas, aunque también púnicas, asociada a la idea difusa de una rápida y completa absorción de las comunidades indígenas por los sistemas productivos introducidos por los colonizadores, limita fuertemente la consideración del ajuar cerámico como expresión de unos gustos alimentarios, modos de consumir las comidas y bebidas y símbolos de la pertenencia a un grupo social y étnico (?). El escaso conocimiento de la vajilla local del Bronce Final, unido a la visión colonialista de las relaciones entre poblaciones indígenas y foráneas, reduce la identificación de formas/funciones tradicionales del mundo local prefenicio. Un ejemplo son los cuencos de labio engrosado que, en tipos evolucionados, encontramos sin interrupción desde el Bronce Final hasta finales del siglo VI / inicios $\mathrm{V}$ a.C. realizados a mano, o a torno en cerámica gris, barniz rojo, polícroma y sin tratamiento, que perduraron durante la II Edad del Hierro. Un mayor conocimiento sobre el repertorio cerámico y las costumbres alimentarias permitiría avanzar diversas hipótesis sobre las adopciones foráneas, las adaptaciones locales 
a un diferente sistema productivo -el horno bicameral junto al torno alfarero- $y$, consecuentemente, a un cambio en la estructura artesanal económica de las poblaciones indígenas y su extensión a nivel local y/o comarcal. En este sentido, en los últimos años se están realizando estudios específicos sobre las dietas de las poblaciones "turdetanas" establecidas en el Bajo Guadalquivir, analizándolas desde diferentes perspectivas: la lectura crítica de las fuentes literarias, los contextos arqueológicos, los datos polínicos y faunísticos (García Fernández y García Vargas 2010; véase para un estudio más exhaustivo sobre la Turdetania y la Bética occidental: García Fernández y García Vargas 2014).

De todas maneras, analizando el horno cerámico de Ronda ciudad como síntoma del contexto geográfico, social y político en el cual se halla, es posible proponer una hipótesis sobre el tipo de sociedad y el sistema productivo implicado. Durante el siglo V a.C., los datos arqueográficos de la Depresión de Ronda muestran la centralización y jerarquización de la sociedad en torno a grupos dominantes que luchan por el poder o deben aparentar su rol político a través de simbologías y códigos de comportamiento, de los cuales la construcción de murallas representa un ejemplo. Este proceso se puede rastrear desde el siglo VI a.C., cuando el asentamiento de Ronda la Vieja será abandonado y se ocupará la cercana Silla del Moro (Carrilero y Aguayo 2008: 188-192; Aguayo y Sanna en prensa) que, fortificada y con una embrionaria planificación urbanística, es probablemente expresión de un nuevo grupo social que busca su legitimación política (?), manteniendo el control del territorio geográfico, pero ocupando simbólicamente otro lugar destacado en el paisaje de la zona (véase Carrilero 2001: 282-283 y su propuesta de "un modelo supraétnico" organizado en torno al hidrónimo Cilbus, el actual Guadalete). Por lo que concierne a Ronda ciudad en el siglo V a.C., hay que reconocer que el mismo tipo de excavación urbana en la cual se ha encontrado el horno, junto con la característica del asentamiento, poblado ininterrumpidamente ocupado desde época prehistórica hasta la actualidad, no han permitido encontrar otras estructuras relacionadas como espacios de decantación, secado, torneado, almacenamiento, etc., pero la hipótesis de que se trate de un taller alfarero está apoyada por otros datos arqueológicos. Es justamente durante el siglo V a.C. -según la comparación de los datos arqueográficos obtenidos en el asentamiento monofásico de Silla del Moro, que se abandona al comienzo de esta centuria, y con la coincidente reocupación del yacimiento de Ronda la Vieja- cuando en los asentamientos de la Serranía se experimentan una serie de cambios en la organización del trazado urbano, con la construcción de calles centrales a las cuales se abren viviendas dispuestas en batería, mientras la zona productiva, donde ha aparecido el horno, es desplazada hacia la periferia del asentamiento. Además tanto Ronda ciudad como Ronda la Vieja, esta última probablemente amurallada en el siglo V a.C., participan activamente en las actividades comerciales impulsadas por los centros gaditanos y los malagueños, como parece atestiguado por la persistencia de una ruta terrestre alternativa al paso marítimo del estrecho de Gibraltar (se ha propuesto la existencia arqueológica de la ruta durante la primera mitad del I milenio a.C. en Aguayo et al. 1995: 85-97; mientras que para su prosecución en la II Edad del Hierro véase: Aguayo y Sanna 2014: 626-627), que atraviesa la Depresión y conecta los dos importantes puertos de Gadir y Malaka. En Ronda la Vieja, y sobre todo en Ronda ciudad, los testimonios fundamentales de esta ruta $\mathrm{y}$ de la presencia de grupos dominantes como principales "clientes" son la vajilla de lujo griega, las cuentas de pasta vítrea, las piezas de marfil y las salazones de pescado que llegan a la comarca.

Finalmente, por lo que concierne a la producción alfarera, podemos observar la presencia de una cierta especialización en los tipos y decoraciones documentadas, lo que nos acerca a la propuesta de E. Ferrer y F. J. García (2008: 202-203) de la presencia de una línea de formación continua entre maestros y aprendices, que garantiza la realización de un ajuar cerámico plenamente funcional para satisfacer las necesidades cotidianas y simbólicas de esta sociedad. Solamente en algunas variantes morfológicas y en el repertorio decorativo se aprecian diferencias que podrían imputarse a los mismos alfareros. De hecho, la producción de cerámicas comunes, funcionales en su forma y repetitivas en los acabados, junto con vajillas que consideramos simbólicas por la complejidad de los motivos decorativos -como queda atestiguado por la pieza RO'89/7546-9 (fig. 4 g)-, nos sugiere la coexistencia de fabricaciones de uso cotidiano con otras dirigidas a un grupo social destacado o a actos rituales específicos, sean ceremonias, banquetes, festividades, etc. La presencia de la cerámica a mano se reduce al mínimo, atestiguada todavía en las formas destinadas a la cocción de alimentos y, posiblemente, a la iluminación -el cuenco-lucerna- o como servicio de mesa, indicando de una parte la coexistencia de dos sistemas productivos, el artesanal y el doméstico, y de otra la permanencia de costumbres alimentarias previas a la II Edad del Hierro. 


\section{ANEXO: EL PROCESO DE RESTAURACIÓN DE LOS MATERIALES CERÁMICOS Y LAS INFERENCIAS CONTEXTUALES ARQUEOLÓGICAS SEGÚN LAS CONDICIONES DE CONSERVACIÓN}

Cuando se manejan materiales que provienen de una excavación arqueológica, con mucha frecuencia, se olvida la importancia de su conservación después que hayan sido sacados del contexto sedimentario (Brandi 1963: 31-40), de cara a las implicaciones interpretativas desde la tecnología de fabricación, usos culinarios o funcionales, las circunstancias de abandono y condiciones de enterramiento, tan fundamentales en lecturas arqueológicas de contextos como espacios cerrados $\mathrm{y}$ con funciones tan singulares como un horno alfarero abandonado y reutilizado. Las observaciones e implicaciones deducibles del manejo, limpieza, consolidación y reconstrucción del material cerámico analizado se han incorporado a los apartados 2 y 3 de este trabajo.

En el caso específico que estamos tratando, ningún ítem arqueológico ha podido ser intervenido inmediatamente y esto ha causado unos cuantos problemas que vamos a analizar.

En primer lugar, como hemos podido ver, es muy interesante valorar el contexto funcional y sedimentario de la excavación. Por su misma naturaleza de horno en desuso, todo el material encontrado en su interior no se hallaba en las mejores condiciones de deposición. Su estado era muy fragmentario y aleatorio y este particular ha afectado también a todo el proceso de limpieza, reconstrucción y conservación. En segundo lugar, la cerámica estaba cubierta de una capa de suciedad superficial aportada por el medio de sedimentación -compuesto por restos terrosos o arcillosos, concreciones de carbonatos $u$ otras sales insolubles incluidas en el tipo de sedimentos y restos constructivos- que, con el paso del tiempo, se ha "fosilizado" sobre el mismo material y que ha sido más difícil de eliminar. En algunos casos que veremos más adelante, esto ha afectado a la lectura contextual y morfológica, así como a la conservación de algunas decoraciones. Finalmente el tiempo que ha pasado entre el momento de la excavación y el análisis del material ha añadido algunas dificultades, ya que los fragmentos se encontraban divididos en lotes de excavación, individualizados en bolsas de plástico, poco porosas, y conservadas en un ambiente escasamente estable. Dichas bolsas han sido revisadas una por una para poder encontrar eventuales correspondencias individuales y formales, tarea que aunque retrasó el trabajo era fundamental tanto para la presumible reconstrucción formal y las características tecnológicas y de conservación, como para las implicaciones estratigráficas y contextuales/funcionales.

Una vez que todos los materiales han sido divididos y fotografiados con su número correspondiente se ha podido empezar el verdadero trabajo de limpieza, análisis y conservación (fig. 5).

El primer paso de la mecánica del laboratorio ha sido una limpieza manual a fondo y cuidadosa para evitar dañar, aunque fuera en mínima parte, las piezas o perder material (Carrascosa 2009: 73-84). Para ello ha sido indispensable el uso de un bisturí de hoja fina que se ha utilizado para la eliminación de las concreciones superficiales de carbonatos. Por esta razón, la primera limpieza superficial en seco ha sido acompañada de una segunda efectuada con agua desmineralizada y un cepillo suave para ablandar y eliminar todos los restos concrecionados. Este proceso ha sido efectuado con el mayor cuidado posible en fragmentos decorados y con restos de pigmentación, para asegurar su perfecta conservación y posterior lectura composicional.

Además, en algunos casos puntuales, la cerámica mostraba concreciones de carbonatos muy duros y resistentes. No obstante como la estabilidad de los carbonatos no afectaba a la conservación en sí misma de estas piezas, se ha procedido a su eliminación con el uso alternativo de un bisturí de hoja fina y otro de hoja larga. Esta eliminación se ha considerado necesaria para garantizar la lectura de las piezas, sobre todo cuando afectaba a fragmentos que mostraban algún tipo de decoración.

De hecho, la pigmentación de las piezas es la que se ha visto más afectada y más deteriorada por la larga falta de conservación y cuidados. En muchos casos hemos podido observar desprendimientos de fragmentos de la capa pictórica, tanto que frecuentemente ha sido necesaria una consolidación antes de proceder a cualquier limpieza, para poder asegurar lo conservado y evitar la pérdida de cualquier información durante la manipulación analítica y de conservación. En este caso la consolidación ha sido efectuada con Primal -pegamento acrílico- en una concentración entre el 5 y el $15 \%$ en agua desmineralizada, según la necesidad (Carrascosa 2009: 113-122).

El proceso de limpieza y, cuando es necesario, la consolidación han sido seguidos por la reunificación de los fragmentos. Este proceso ha sido muy complicado por varias razones: en primer lugar por el mismo hecho de que se trataba del vaciado por excavación de un horno abandonado y, por lo tanto, los materiales 

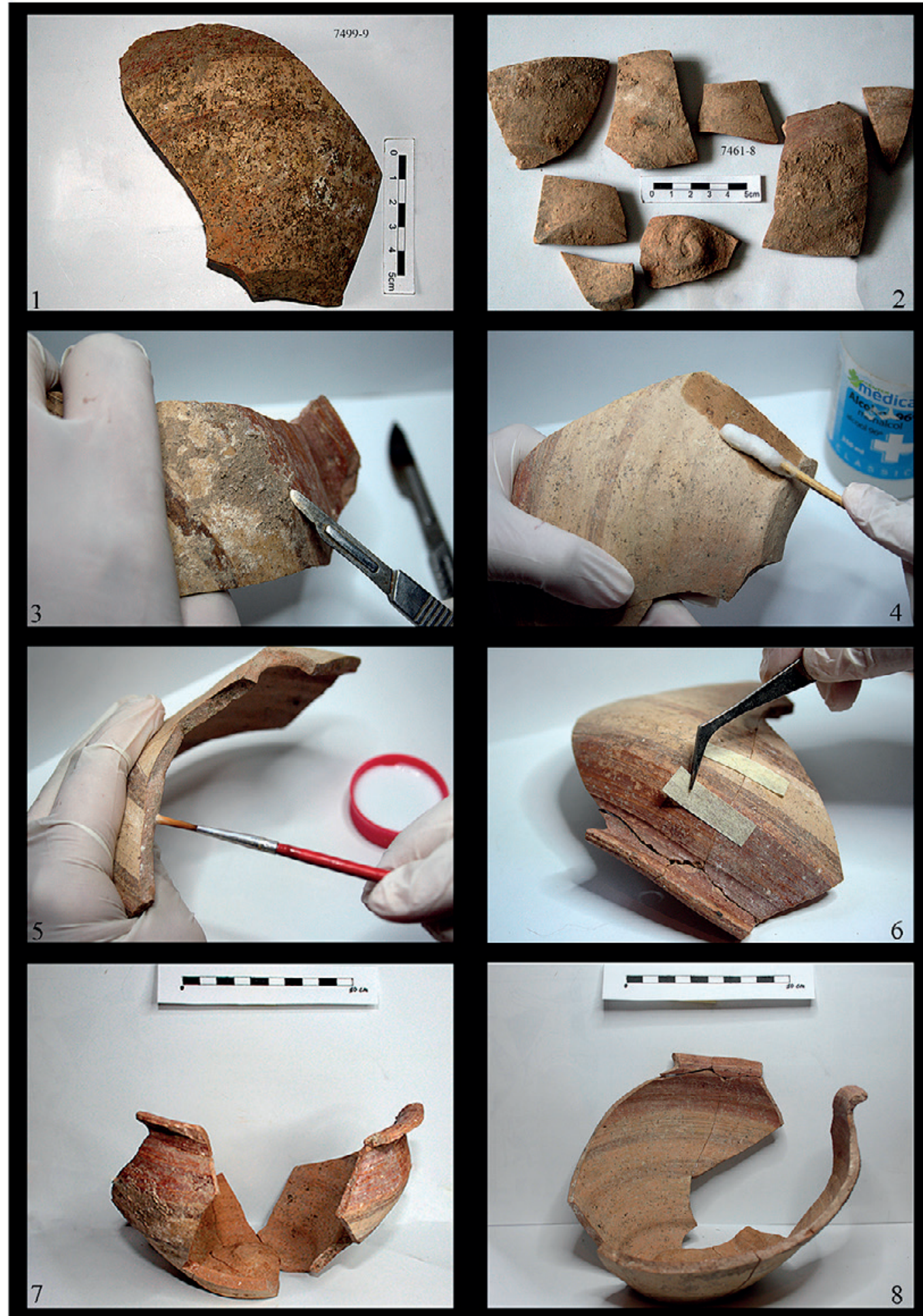

Figura. 5. Historia fotográfica de las intervenciones en la pieza RO'89-AA/7461-8 y 7499-9. 1-2 Fragmentos antes de cualquier intervención; 3. Limpieza manual con bisturí; 4. Limpieza manual con alcohol; 5. Pegado con cola vinílica; 6. Secado del pegamento. La cinta es indispensable para mantener en posición los fragmentos; 7-8. Pieza restaurada. 
eran, en su totalidad, desechos; en segundo lugar por el estado fragmentario del material; y finalmente por la dificultad de encontrar correspondencias entre los diferentes fragmentos agrupados por unidades de excavación en diversos lotes de material arqueológico.

Una vez que los diversos ítems han sido reconocidos se ha decidido proceder, cuando posible, a su reconstrucción. Nuestra elección ha sido una cola vinílica disuelta en agua desmineralizada, con gran capacidad de penetración en el material poroso y un secado lento, que permite rectificar posibles errores de posición y eliminar fácilmente el exceso con algodón y agua.

Finalmente, una vez que los fragmentos han sido reconocidos, recomponiéndolos cuando ha sido posible, nos hemos encontrado con una nueva decisión a tomar en nuestro trabajo de conservación y análisis del material cerámico del horno de la ciudad de Ronda: ¿qué hacer con las piezas cuando ha sido posible su reconstrucción aunque fuera parcial? Se ha optado por no restituir las piezas con añadidos de yeso o escayola, que añadirían tensiones innecesarias a un material tan frágil, no siendo imprescindible la reconstrucción ni para la lectura arqueológica ni para la eventual exposición museística.

\section{Agradecimientos}

La excavación, bajo la modalidad administrativa de Urgencia, en aplicación de las cautelas arqueológicas impuestas por la Comisión Local del Patrimonio Histórico Artístico de Ronda, se llevó a cabo en las calles Armiñán no 39, 41, 43 y Aurora n 16, en el marco del proyecto "Arqueología Urbana de la Ciudad de Ronda”, codirigido por M. Carrilero, B. Padial y O. Garrido, y autorizado por la Dirección General de Bienes Culturales de la Consejería de Cultura de la Junta de Andalucía. La excavación ha sido subvencionada por el promotor de la promoción inmobiliaria.

Agradecemos al Prof. Pedro Aguayo de Hoyos, profesor del Departamento de Prehistoria y Arqueología de la Universidad de Granada, por facilitarnos toda la documentación relativa al horno bicameral de Ronda ciudad, incluido tanto el material cerámico estudiado como las plantas y el inventario de los materiales. Además, le agradecemos sus reflexiones expuestas a lo largo de todo el proceso de escritura de este artículo.

Agradecemos al Prof. Andrés M. Adroher Auroux, profesor del Departamento de Prehistoria y Arqueología de la Universidad de Granada, por las indicaciones cronológicas y por sus aportaciones a la interpretación del material contenido en el horno alfarero.

\section{BIBLIOGRAFÍA}

Aguayo, P. y Sanna, C. (2014): “Considerazioni sul rinvenimento di ceramica d'importazione pre-romana negli insediamenti dell'hinterland del Circolo dello Stretto", en Rei Cretariae Romanae Fautorum Acta 43: Congressus vicesimus octavus Rei Cretariae Romanae Fautorum Catinae habitus MMXII. Actas del $28^{\text {th }}$ Congress of the Rei Cretariae Romanae Fautores: 623-630. Catania (2012), Bonn, Freiburger graphische betriebe (FGB) $\mathrm{GmbH} \& \mathrm{Co}$. KG.

Aguayo, P.; Castilla, J. y Padial, B. (1992): "Excavación de urgencia en el casco antiguo de Ronda. Calle Armiñán no 39, 41, 43 y Aurora no 16. 1989”. Anuario Arqueológico de Andalucía 1990, Tomo III: 339-342. Sevilla, Consejería de Cultura, Junta de Andalucía.

Aguayo, P.; Garrido O. y Padial, B. (1995): "Una ruta terrestre alternativa al Paso del Estrecho en época orientalizante: constatación arqueológica", en Arqueología Clásica e Historia Antigua. Actas del II Congreso Internacional «El Estrecho de Gibraltar», Tomo II: 85-97. Ceuta (1990), Madrid, UNED.

Aguayo, P.; Sanna, C. y Padial, B. (2013): "Documentos para ilustrar una tradición alfarera local: un horno cerámico ibérico en Ronda ciudad", en D. Bernal, L.C. Juan, M. Bustamante, J.J. Díaz y A.M. Sáez (eds.), Hornos, talleres y focos de producción alfarera en Hispania, Monografías Ex Officina Hispana 1, Tomo I: 141-156. Cádiz, Universidad de Cádiz.

Aubet, M.E.; Carmona, P.; Curià, E.; Delgado, A.; Fernández Cantos, A. y Párraga, M. (1999): Cerro del Villar - I. El asentamiento fenicio en la desembocadura del río Guadalhorce y su interacción con el hinterland. Monografias Arqueología 5. Sevilla, Junta de Andalucía.

Belén Deamos, M. (2007): “Ánforas de los siglos VIIV a.C. en Turdetania”. Spal 15: 217-246. http://institucional.us.es/revistas/spal/15/art_11.pdf

Brandi, C. (1963): Teoria del Restauro. Roma, Edizioni di Storia e Letteratura.

Carrascosa, B. (2009): La conservación y restauración de objetos cerámicos arqueológicos. Madrid, Tecnos.

Carrilero, M. (1992): "El proceso de transformación de las sociedades indígenas de la periferia tartésica", en J. L. López Castro (ed.), La colonización fenicia en el Sur de la Península Ibérica. 100 años de Investigación: 117-142. Almería, Instituto de Estudios Almerienses y Facultad de Humanidades de Almería. 
Carrilero, M. (2001): "El comercio ibérico del siglo VI al III a.C.", en Comercio y comerciantes en la Historia Antigua de Málaga (siglo VIII a.C.-año 711 d.C.). Actas del II Congreso de Historia Antigua de Málaga: 277-297. Málaga (1998), Málaga, Diputación Provincial de Málaga.

Carrilero, M. y Aguayo, P. (2008): "Entre Tartesios y Turdetanos, entre el Bajo Guadalquivir y las Béticas Occidentales, entre los Siglos VII y V a.C.: ¿Cilbicenos?”, en A.M. Adroher Auroux y J. Blánquez Pérez (eds.), I Congreso Internacional de Arqueología Ibérica Bastetana. Serie Varia 9: 179195. Madrid, Universidad Autónoma de Madrid, Universidad de Granada y Asociación de Estudios de la Arqueología Bastetana.

Escacena Carrasco, J.L. (1979-1980): “Cerámica ibérica de Setefilla (Sevilla)”. Pyrenae 15-16: 181-210.

Escacena Carrasco, J.L. (1989): "Los turdetanos o la recuperación de la identidad perdida”, en M.E. Aubet (coord.), Tartessos. Arqueología Protohistórica del Bajo Guadalquivir: 433-476. Sabadell, Ausa.

Escacena Carrasco, J.L. (1992): "Indicadores étnicos en la Andalucía prerromana”. Spal 1: 321-343. https:// idus.us.es/xmlui/bitstream/handle/11441/13960/ file_1.pdf?sequence $=1$

Escacena Carrasco, J.L. (2001): "Podando a Carmo. Perfiles del sustrato turdetano", en A. Caballos Rufino (ed.), Carmona Romana. II Congreso de Historia de Carmona: 21-35. Carmona (1999). Sevilla, Universidad de Sevilla.

Ferrer, E. y García Fernández, F.J. (2002): “Turdetania y Turdetanos: Contribución a una Problemática Historiográfica y Arqueológica". Mainake XXIV: 133-151.

Ferrer, E. y García Fernández, F.J (2008): “Cerámica turdetana”, en D. Bernal Casasola y A. Ribera i Lacomba (eds.), Cerámicas hispanorromanas. Un estado de la cuestión: 201-219. Cádiz, Servicio de Publicaciones de la Universidad de Cádiz con la colaboración de la Fundación Pouroulis.

García Fernández, F.J. (2012): “Tartesios, Túrdulos, Turdetanos. Realidad y Ficción de la Homogeneidad Étnica de la Bética Romana”, en J. Santos y G. Cruz (eds.), M. Fernández y L. Sánchez (cols.), Revisiones de Historia Antigua VII. Romanización, fronteras y etnias en la Roma antigua: el caso hispano: 691-734. Vitoria-Gasteiz, Argitalpen Zerbitzua.

García Fernández, F.J. y García Vargas, E. (2010): “Entre gaditanización y romanización: Repertorios cerámicos, alimentación e integración cultural en Turdetania (siglos III-I a.C.)", en C. Mata, G. Pérez y
J. Vives-Ferrándiz (eds.), De la Cuina a la Taula. IV Reunió d'economia en el Primer Mil.leni a.C. Saguntum EXTRA 9: 115-134. Valencia, Universidad de Valencia.

García Fernández, F.J. y García Vargas, E. (2012): “Los hornos alfareros de tradición fenicia en el Valle del Guadalquivir y su perduración en época romana: aspectos tecnológicos y sociales". Spal 21: 9-39. http://institucional.us.es/revistas/spal/21/art_1.pdf

García Fernández, F.J. y García Vargas, E. (2014): Comer a la moda. Imitaciones de vajilla de mesa en Turdetania y la Bética Occidental durante la Antigüedad (S. VI a.C. - VI d.C.). Col·lecció Instrumenta 46. Barcelona, Universidad de Barcelona.

Mata, C. y Bonet, H. (1992): "La cerámica ibérica: ensayo de tipología", en Estudios de Arqueología ibérica y romana. Homenaje a Enrique Pla Ballester. Servicio de investigación prehistórica. Serie de trabajos varios 89: 117-173. Valencia, Diputación Provincial de Valencia.

Munsell (1994): Soil Color Charts. Revised Edition. New York, Munsell Publishing Company.

Orton, C.; Tyers, P. y Vince, A. (1997): Cerámica en Arqueología. Barcelona, Crítica.

Pellicer, M. (1978): “Tipología y cronología de las ánforas prerromanas del Guadalquivir, según el Cerro Macareno (Sevilla)". Habis 9: 365-400.

Pereira, J. (1988): "La cerámica ibérica de la cuenca del Guadalquivir. I. Propuesta de clasificación". Trabajos de Prehistoria 45: 143-173.

Pereira, J. (1989): "La cerámica ibérica de la cuenca del Guadalquivir. II. Conclusiones". Trabajos de Prehistoria 46: 149-159.

Ramón, J. (1995): Las ánforas fenicio-púnicas del Mediterráneo central y occidental. Col·lecció Instrumenta 2. Barcelona, Universidad de Barcelona.

Ramón, J.; Sáez Espligares, A.; Sáez Romero, A.M. y Muñoz, Á (2007): El taller alfarero tardoarcaico de Camposoto (San Fernando, Cádiz). Monografías Arqueología 26. Sevilla, Junta de Andalucía.

Sáez Romero, A. (2013): “Talleres cerámicos en Gadir en época postcolonial. ¿Un modelo alfarero excepcional?", en D. Bernal, L.C. Juan, M. Bustamante, J.J. Díaz y A.M. Sáez (eds.), Hornos, talleres y focos de producción alfarera en Hispania, Monografías Ex Officina Hispana 1, Tomo I: 215-249. Cádiz, Universidad de Cádiz.

Sanna, C. y Aguayo, P.: "Las Béticas Occidentales y el problema de sus inclusiones en la Turdetania del siglo V a.C." (en prensa): en La Sardegna nel Mediterraneo occidentale dalla fase fenicia all'egemonia 
cartaginese: il problema del V secolo. Atti del Convegno Internazionale di Studi. Santadi (2013).

Thiriot, J. (1992): “Du four a l'atelier de potier: quelques reflexions pour une meilleure connaissance de cet artisanat medieval", en Tecnología de la cocción cerámica desde la antigüedad a nuestros días. Ponencias del Seminario celebrado en el Museo de Alfarería en Agost (Alicante) del 4 al 6 de octubre de 1990: 140-152. Agost (Alicante) (1990). Alicante, Asociación de Ceramología. 